\title{
The formation and stability of recognition memory: what happens upon recall?
}

\author{
Sabrina Davis ${ }^{1,2}$, Sophie Renaudineau ${ }^{3}$, Roseline Poirier ${ }^{1,2}$, Bruno Poucet ${ }^{3}$, Etienne Save ${ }^{3}$ and Serge Laroche ${ }^{1,2 *}$ \\ Centre de Neurosciences Paris-Sud, UMR 8195, Univ Paris-Sud, Orsay, France \\ 2 CNRS, UMR 8195, Orsay, France \\ ${ }^{3}$ Laboratoire de Neurobiologie de la Cognition, UMR 6155, CNRS, Aix-Marseille University, Marseille, France
}

\section{Edited by:}

Jacek Debiec, New York University,

USA

\section{Reviewed by:}

Jacek Debiec, New York University, USA

Phillip R. Zoladz, Ohio Northern

University, USA

*Correspondence:

Serge Laroche, Centre de

Neurosciences Paris-Sud, CNRS, UMR

8195, University Paris-Sud, F-91405

Orsay, France.

e-mail: serge.laroche@u-psud.fr
The idea that an already consolidated memory can become destabilized after recall and requires a process of reconsolidation to maintain it for subsequent use has gained much credence over the past decade. Experimental studies in rodents have shown pharmacological, genetic, or injurious manipulation at the time of memory reactivation can disrupt the already consolidated memory. Despite the force of experimental data showing this phenomenon, a number of questions have remained unanswered and no consensus has emerged as to the conditions under which a memory can be disrupted following reactivation. To date most rodent studies of reconsolidation are based on negatively reinforced memories, in particular fear-associated memories, while the storage and stability of forms of memory that do not rely on explicit reinforcement have been less often studied. In this review, we focus on recognition memory, a paradigm widely used in humans to probe declarative memory. We briefly outline recent advances in our understanding of the processes and brain circuits involved in recognition memory and review the evidence that recognition memory can undergo reconsolidation upon reactivation. We also review recent findings suggesting that some molecular mechanisms underlying consolidation of recognition memory are similarly recruited after recall to ensure memory stability, while others are more specifically engaged in consolidation or reconsolidation. Finally, we provide novel data on the role of Rsk2, a mental retardation gene, and of the transcription factor zif268/egr1 in reconsolidation of object-location memory, and offer suggestions as to how assessing the activation of certain molecular mechanisms following recall in recognition memory may help understand the relative importance of different aspects of remodeling or updating long-lasting memories.

Keywords: recognition memory, object memory, consolidation, reconsolidation, knockout mice, Rsk2, zif268/egr1, Coffin Lowry

\section{INTRODUCTION}

The notion that an already consolidated memory could be destabilized and disrupted was first demonstrated in the late 1960s by Lewis and colleagues (Misanin et al., 1968) amidst an environment where the prevailing theory at the time, the consolidation theory, suggested that once a memory was consolidated, it was permanent or fixed. This was based on a dichotomy between short and longterm memory and it was assumed, though far from being proven, that amnesia was a permanent consolidation or retrieval deficit. It was against this backdrop that Lewis argued, it was more parsimonious to consider that memory traces, regardless of their age, could shift between two states, an inactive and stable (consolidated) state corresponding to stored memories and an active and fragile state "open to disruption by amnestic agents" following the encoding of information and the readout of the trace during retrieval (Lewis, 1979). The implication is that when the memory is converted again into an active state following reactivation, a further storage process is required for the trace to remain in long-term memory and be available once again for recall. This was based on his own studies and number of others that showed a so-called consolidated memory could be disrupted when electroconvulsive shock (Misanin et al., 1968), or interfering material (Gordon and Spear, 1973) were given shortly after a memory was reactivated or recalled. Despite this, Lewis' experiments and arguments went largely unheeded for some 20-30 years, as the consolidation theory remained doggedly in place until Przybyslawski and Sara (1997) and Nader et al. (2000) resurrected Lewis' theory. Nader et al. (2000) showed that a "consolidated" fear memory could be disrupted by the protein synthesis inhibitor, anisomycin if injected just after reactivation of the memory, $24 \mathrm{~h}$ following initial learning. Importantly, they showed that if protein synthesis inhibition occurred in the absence of reactivation of the memory, it remained intact.

Subsequent to this initial resurrection of Lewis' results, there followed a slew experiments replicating Nader and colleagues basic findings in a wide range of species from nematode (Rose and Rankin, 2006) to honey bees (Stollhoff et al., 2005), sea slug (Sangha et al., 2003), rodents (Nader et al., 2000; Kida et al., 2002; Milekic and Alberini, 2002; Suzuki et al., 2004; see reviews by Hardt et al., 2010; Nader and Einarsson, 2010) through to human studies (Walker et al., 2003; Tronson and Taylor, 2007; Hupbach et al., 2009; Schwabe and Wolf, 2009). This reinforced the notion that memories were not "fixed" or permanent and when in an active state they were labile and susceptible to disruption by interfering events to a level where the scientific community accepted that this was now a bone fide phenomenon, common across species (see Nader and Einarsson, 2010 for details on supporting experimental data). 
Despite the overwhelming support for reconsolidation there were the inevitable studies that showed an exception to the rule and a number of what was termed "boundary conditions" were applied in circumstances where a reactivated memory remained resistant to disruption, and therefore required no need for reconsolidation. To date the most commonly encountered boundary conditions that determine whether reactivated memories are vulnerable or immune to disruption are the age of the memory (Milekic and Alberini, 2002; Suzuki et al., 2004; Robinson and Franklin, 2010), the strength of learning (Suzuki et al., 2004; Taylor et al., 2009), duration of stimuli during reactivation (Suzuki et al., 2004; Power et al., 2006) and the degree of similarity between the environment and conditions in which the memory was consolidated and reactivated (Bozon et al., 2003a; Debiec et al., 2006; Artinian et al., 2007; Winocur et al., 2009).

The fact that boundary conditions exist, itself raised the question of whether or not reconsolidation was truly a re-enactment of the consolidation process. This question has largely evolved from studies designed to understand the cellular and molecular mechanisms associated with consolidation and reconsolidation. A number of studies using inhibitors of de novo protein synthesis have shown that new proteins are necessary for both the initial encoding of a memory and its re-stabilization after reactivation, which would suggest reconsolidation is a faithful recapitulation of consolidation. It is not surprising that protein synthesis inhibitors impair both processes, as in order to have any effect on memory, nearly $90 \%$ of all proteins must be inhibited; therefore, at least in terms of understanding the cellular and molecular mechanisms associated with both processes, the use of protein synthesis inhibitors lends little information about the specific genes and proteins that may be involved. In those studies targeting specific genes and proteins there are variations on whether activation of the same proteins and genes are necessary for both processes or whether they are involved in one and not the other. There are a number of studies that have shown a given gene or protein is expressed/ activated in both processes or that selective knockdown of a gene or inhibition of a protein impairs both processes (reviewed in Tronson and Taylor, 2007). However, dissociations have also been shown; for example, in a fear conditioning task, it was shown, using oligonucleotide antisense, that regulation of the transcription factor, Zif268/Egr1 in CA1 of the hippocampus was not necessary for consolidating fear memory, but was necessary for reconsolidation of the active memory (Lee et al., 2004). Conversely, other studies have shown the transcription factor CCAAT/enhancer binding protein $\mathrm{C} / \mathrm{EBP} \beta$ is activated and necessary in the dorsal hippocampus in association with consolidation of fear memory but not its reconsolidation (Taubenfeld et al., 2001). And yet another variation on the theme is the demonstration that consolidation and reconsolidation may activate the same genes and proteins but in different neural circuits (Kelly et al., 2003; Alberini, 2005). This suggests for certain memories, restabilizing the memory after reactivation is not a simple reiteration of the consolidation process, at least in terms of the underlying mechanisms. This is also supported by data showing that the memory trace takes a shorter time to restabilize than it does to consolidate (Litvin and Anokhin, 2000; Milekic and Alberini, 2002; Suzuki et al., 2004; Languille et al., 2009). However, there is controversy as to whether consolidation and reconsolidation are separate but overlapping processes (Nader et al., 2000; Debiec et al., 2002; Nader and Hardt, 2009) or whether in fact it is merely part of an extended and dynamic form of consolidation (Dudai and Eisenberg, 2004; Alberini, 2005) that has just not yet been experimentally demonstrated. This was an issue raised a number of years ago and appears to date not to be resolved. Whether reconsolidation is an independent or even partially independent process or whether it is part of an ongoing consolidation process, whether the same molecules are required or not, whether the same circuitry is involved or not, are among the major issues debated in the field. As it is generally agreed that memories are encoded in widespread neural networks and neuropsychological evidence suggests that memories are encoded over lengthy periods of time, it would seem inevitable that when a memory is reactivated it is available for some form of modification and its content could well evolve over time depending on the environment and circumstances in which it is reactivated. It therefore may be fruitful to use the cellular and molecular mechanisms that have been associated with consolidation and reconsolidation to understand how memories evolve over time following repeated reactivation.

A second, equally controversial debate that is intertwined with the dispute as to whether reconsolidation is a separate entity or part on a slow process of consolidation concerns the function of reconsolidation. In other words, why each time a memory is reactivated it is vulnerable to loss or disruption and requires reconstruction, as this is a costly and somewhat dangerous mechanism. Currently there are two principle theories as to the functional role of reconsolidation that suggest it is either a means of strengthening the memory trace (Alberini, 2005) or that it is a mechanism whereby the memory trace may be updated to incorporate new information (Morris et al., 2006; Hupbach et al., 2007; Lee, 2009) neither of which seems to be mutually exclusive of each other. Implicit in the theory that reconsolidation serves to strengthen memory is the notion that eventually memories should become immune to disruption. Those that support this theory offer evidence that suggests newer memories are more vulnerable to disruption following reactivation than older memories (Alberini, 2005; Wang et al., 2009). Furthermore, they suggest that strengthening the memory via a mechanism such as reconsolidation may be a means of explaining addictive behavior and pathological memories associated with post-traumatic stress disorder (PTSD) and phobias. Indeed, at the clinical level, as behavioral therapy using extinction to attempt to eliminate or decrease episodes of stress associated with these pathological memories have not met with a great deal of success, the emphasis has changed to attempt to disrupt types of memories by reactivating them and administering drugs associated with mechanisms known to disrupt reconsolidation. At present, however, it is not clear what rate of success these treatments have had. A recent experiment in human subjects has shown that a form of fear conditioning with mild shock to the wrist can be erased if the memory is reactivated just prior to an extinction protocol that is successful a year later (Schiller et al., 2010). However, given the strength of the emotional content and the complexity of pathological memories in phobias or drug addiction or PTSD, whether simple behavioral modification such as this would constitute an effective treatment for eliminating these types of memory or whether it could work successfully with older memories is not clear. 
Opposed to the memory strengthening theory of reconsolidation is that its function is to update memories to integrate new information to confer adaptive benefit to the organism. It has been shown in both rodents and humans that subsequent additional learning can modify the original memory if it is in an active state. A number of studies using animals and humans have suggested that subsequent additional learning can modify the original memory (Hupbach et al., 2009; Lee, 2009; Schiller et al., 2010). An important feature of this theory is the implication that regardless of the age of the memory, it can be altered and that at a systems level of consolidation or reconsolidation, memories can be linked to form a knowledge base in much the same manner of Bartlett's schemata (see Hardt et al., 2010). Although most of the experimental evidence to suggest the fluidity of memory and remembering has been within the domain of human studies, Tse et al. (2007) have demonstrated that rats can rapidly incorporate new taste-spatial information into an existing memory. The importance of this theory and the evidence that supports it afford us an inroad into an area of research that has largely remained out with behavioral studies and the neurosciences to understand how memories can be built up and elaborated over time.

To date, most rodent studies aimed at investigating the cellular and molecular mechanisms associated with reconsolidation have used negatively reinforced learning tasks such as fear conditioning or spatial learning in the water maze. Relatively few studies have used non-emotive learning, and fear learning may constitute a highly specific form of learning in which important but subtle changes in the memory may not be detectable. In this review, we focus on recognition memory, a form of memory that can be based on incidental encoding, not relying on any explicit reinforcement. Recognition memory is highly relevant to all species, it plays a key role in adaptive behaviors and is implicated in all forms of memory regardless of whether they are or not emotionally based, negatively, or positively reinforced.

\section{MEMORY PROCESSES AND NEURAL CIRCUITS ENGAGED IN RECOGNITION MEMORY}

Recognition memory is a prototypical form of event memory widely used in specific paradigms to probe episodic memory in humans. Operationally, it refers to the ability to recognize previously encountered information and is commonly defined in humans as the conscious recollection of past personally experienced events (Tulving, 2002). The most common paradigms used for testing recognition memory in human subjects involve verbal material or visuospatial recognition of faces, objects, or scenes. Performance in recognition memory retrieval can however be based on at least two functionally distinct memory processes: it can rely on familiarity detection, a mental awareness that an event has been experienced in the past, leading to a sense of basic "feeling of knowing" or "déjà vu" which is experienced rapidly, or on genuine recollection, the conscious remembering of the event incorporating detailed episodic features such as the content and spatio-temporal context within which the event occurred. These two processes are not mutually exclusive and boundaries between them are hazy, as on one side elements of familiarity are likely to occur in all recall tasks and on the other performance does not always provide insight into retrieval accuracy and confidence of recollection.
Several studies examining recognition memory performance in amnesic patients have revealed deficits in patients suffering medial temporal lobe lesion. Although both recollection and familiarity detection can be affected by medial temporal lobe lesion, recollection is often more severely impaired than familiarity (reviewed in Eichenbaum et al., 2007) and whereas several studies support the idea that recollection engages the hippocampus, the parahippocampal region can support familiarity (Brown and Aggleton, 2001; Eichenbaum et al., 2007). Attempts to delineate the neural circuits that underlie the two processes at retrieval using electrophysiological or neuroimaging techniques in humans also support the idea that these two processes engage at least partly distinct brain systems. For example, event-related potentials recordings in tasks that allow the dissociation between true recollection and levels of familiarity of retrieved items revealed a mid-frontal negativity which onsets at $\approx 300 \mathrm{~ms}$ and varies with familiarity strength, while recollected items are associated with a left parietal positivity, insensitive to familiarity, with a more delayed onset around $500 \mathrm{~ms}$ (Woodruff et al., 2006; see Rugg and Curran, 2007 for a review). Using graded confidence ratings, Yonelinas et al. (2005) described two non-overlapping circuits involved in familiarity strength and recollection judged by recall of thoughts about the words and their shape on the screen. Using fMRI, they highlight a circuit involving areas of the lateral and medial (precuneus) parietal and of the anterior and lateral prefrontal cortices activated in relation to increased confidence in familiarity, whereas recollection-based recognition is associated with activation of distinct areas of the anterior medial prefrontal, posterior cingulate, lateral parietal and temporal cortices, and the hippocampus. As a further example, a study exploring the effect of sub-seizure electrical stimulation of the hippocampus during recognition memory tasks in humans reported deficits in word recognition after left hippocampal stimulation and deficits in face recognition after right hippocampal stimulation (Coleshill et al., 2004). These and other studies thus support the notion that recollection engages a network of structures that includes the hippocampus, while familiarity detection even with a high level of confidence is less consistently associated with hippocampal activation. Consistent with this, several other imaging studies have reported activation of the perirhinal cortex in association with familiarity and activation of the hippocampus and parahippocampal area in association with recollection-based recognition, although certain studies reported a decrease in activity in these two regions can be associated with familiarity while others suggested there is not a strict dichotomy between circuits of the medial temporal lobe engaged in recollection and familiarity (reviewed in Eichenbaum et al., 2007; Skinner and Fernandes, 2007; Squire et al., 2007).

\section{OBJECT AND OBJECT-PLACE RECOGNITION IN RODENTS}

In rodents it is notoriously difficult to dissociate recollection from familiarity because it is impracticable to measure explicit recall of past experience in animals without language. Recognition memory can however be tested in a task based on the spontaneous preference of rodents for novelty and their ability to remember previously encountered objects (Ennaceur and Delacour, 1988). In the standard protocol, animals are briefly exposed to two or three objects and a memory test can be given after a short or a long time interval by changing one object for a novel object or 
by changing the spatial position of one familiar object. During the test, preferential exploration of the novel or displaced object provides a measure of recognition memory. These tasks are based on incidental encoding during objects sampling, suggesting that performance at retrieval rely on both item familiarity and event recollection (Nemanic et al., 2004). A large body of literature has examined the contribution of the hippocampus and medial temporal lobe structures in object recognition memory in rodents. In early behavioral studies, recognition memory at relatively short delays $(\leq 4 \mathrm{~h}$ ) was shown to be impaired by perirhinal lesions but spared by lesions of the hippocampus (see Mumby, 2001; Winters et al., 2008 for reviews). Conversely, several lines of evidence have pointed to a delay-dependent impairment in long-term $(\geq 24 \mathrm{~h})$ recognition memory in rats with hippocampal lesions (Vnek and Rothblat, 1996; Clark et al., 2000; Hammond et al., 2004; de Lima et al., 2006), depending on lesion size (Broadbent et al., 2004), or in mice with lidocaine-induced inactivation of the hippocampus (Hammond et al., 2004). As another example, post-training lesion of the hippocampus produces retrograde, although not anterograde, amnesia in recognition memory, suggesting the hippocampus, when functional, participates in the formation of recognition memory (Broadbent et al., 2009). These studies, as well as others based on hippocampal specific gene inactivation (see below), lend support to the conclusion that while the perirhinal cortex is critically involved in object recognition (reviewed in Winters et al., 2008), the contribution of the hippocampus becomes increasingly vital with increases in task demand. This depends on several factors such as the size and shape of the testing apparatus, the number and complexity of the objects in the sample phase, the retention delay (Hammond et al., 2004) and the availability and richness of contextual information (see Winters et al., 2008) and their consequences on the ability to form spatial configurations, associative relationships between items and objects-scene relationships (Jenkins et al., 2004). This structural double-dissociation effect seems to parallel the familiarity/recollection dual-process account of recognition memory studied in humans, and is also consistent with several electrophysiological recording studies showing perirhinal cortex neuron correlates of familiarity (reviewed in Winters et al., 2008) and hippocampal neurons correlates of both objects location and identity (Manns and Eichenbaum, 2009). Although there are few tangible means of experimentally testing this in rodents, a recent study using an odor-digging medium based associative recognition paradigm in rats provided striking support to the idea that familiarity detection and recollection are qualitatively dissociable processes and that the hippocampus supports recollection and not familiarity (Sauvage et al., 2008).

\section{CELLULAR AND MOLECULAR MECHANISMS OF RECOGNITION MEMORY CONSOLIDATION}

To date it is largely believed that memory formation involves longlasting, activity-dependent changes in synaptic strength within the neural networks activated during learning, as exemplified by LTP-like mechanisms, and that this is mediated by molecular mechanisms in neurons underlying functional and structural remodeling of network connectivity. The prevailing model for the mechanisms underlying synaptic plasticity and the laying down of memories involves activation of specific cell-surface receptors and of several signaling cascades via kinase pathways that ultimately lead to post-translational modifications of numerous synaptic proteins and to the activation of nuclear transcription factors to trigger the expression of specific gene programs in neurons and the synthesis of proteins. In recent years, a wealth of experimental data has provided compelling evidence that canonical cellular and molecular mechanisms engaged in the formation of most forms of long-term memory are similarly implicated in the consolidation of recognition memory. The most familiar of such example is the requirement of protein synthesis for the stabilization of long-term memory, as demonstrated by the impairment of long-term, but not short-term recognition memory by protein synthesis inhibition in area CA1 of the hippocampus (Rossato et al., 2007). Other experiments however have shown a particular sensitivity to hippocampal protein synthesis inhibition when contextual information is a crucial cue (Balderas et al., 2008). Among the signaling cascades that have been consistently shown to be critical for synaptic plasticity and for the consolidation of several forms of memory, the MAP kinase (MAPK/ERK) cascade has attracted much attention as a key effector of the regulation of gene expression in response to neuronal activation (Sweatt, 2001; Davis and Laroche, 2006 for reviews). Examining whether the MAPK/ERK cascade is also required for the consolidation of object recognition memory, we used a paradigm that most probably places a high demand on hippocampal function by using complex three-dimensional objects in a wide open-field surrounded by rich contextual information and found that object exploration induces rapid phosphorylation of MAPK/ERK in the entorhinal cortex, dentate gyrus and to a lesser extent in CA1 (where novelty seems to be a more efficient activating factor), and that blocking MAPK/ERK phosphorylation during object exploration suppresses long-term, but not shortterm, recognition memory (Kelly et al., 2003). The MAPK/ERK cascade is known to convey signals from cell-surface receptors to the nucleus via transcription factors such as CREB and Elk-1 and to play a critical role in triggering gene expression by activating various inducible nuclear transcription factors (Davis et al., 2000; Waltereit et al., 2001). One such transcription factor is zif268/ egr1, a member of the Egr family of transcriptional regulators that is rapidly activated in a structure dependent manner after different types of learning. Examining key components of this pathway, we found that forebrain expression of a CREB repressor in a transgenic mouse impairs both object and object-location recognition memory (Bozon et al., 2003b). CREB inactivation specifically in area CA1 of the hippocampus was also shown to impair object recognition memory (Pittenger et al., 2002). Similar results were obtained in zif268 mutant mice showing that zif 268 is required for the consolidation of both object and object-location recognition memory (Jones et al., 2001). Zif268 protein is rapidly induced in the dentate gyrus of the hippocampus after object sampling (Soulé et al., 2008) and in mutant mice a gene-dosage effect was found as half the complement of zif268 in heterozygous mutant mice resulted in deficits in long-term object-location memory without impairment in novel object recognition (Bozon et al., 2002). These findings support a model in which a MAPK/ERK-CREBZif268 cascade mediating gene regulation contributes critically to the consolidation of long-term object recognition memory. Surprisingly, other members of the Egr family of transcription 
factors, despite having high homology, conserved DNA binding domains and similar brain localization, were found to affect differentially recognition memory (reviewed in Poirier et al., 2008). While zif268/egr1 deletion affects long-term recognition memory, egr3 deletion affects short-term recognition memory ( $\mathrm{Li}$ et al., 2007) and we found that forebrain-specific deletion of egr2 in transgenic mice facilitates long-term recognition memory (Poirier et al., 2007a), suggesting that the three Egrs do not share similar function and can in contrast have opposite roles in recognition memory, egr2 acting as an inhibitory constrain on recognition memory.

The MAPK/ERK cascade is not the only signaling cascade implicated in recognition memory and over the past several years a collection of studies has provided evidence that recognition memory involves rapid and coordinate regulation of several plasticity-related genes and molecules. For example, object exploration also increases Arc, CaMKII and PSD95 expression in the dentate gyrus (Soulé et al., 2008) and both Arc and CaMKII inactivation in mutant mice have deficient object recognition memory (Miller et al., 2002; Plath et al., 2006). Signaling via type-1 adenylyl cyclases (Wang et al.2004) and via the PI3K-Akt pathway (Horwood et al., 2006) and one of its downstream target mTOR involved in mRNA translation (Myskiw et al., 2008), as well as NMDA and AMPA receptors and several neuromodulators (reviewed in Dere et al., 2007) have also been implicated in object recognition memory. Conversely, forebrain expression of a calcineurin phosphatase inhibitor as well as nuclear protein phosphatase 1 inhibition in transgenic mice were both shown to enhance long-term object recognition memory (Malleret et al., 2001; Koshibu et al., 2009). Epigenetic regulation involving chromatin remodeling through histone post-translational modifications and DNA methylation has also been implicated as a mechanism for gene transcription in recognition memory. For example, histone post-translational modifications at the CREB promoter, were observed after object exploration (Koshibu et al., 2009). The histone acetyltranferase CREB binding protein CBP is required for recognition memory (Barrett and Wood, 2008 for a review) and histone deacetylase inhibition enhances long-term recognition memory (Stefanko et al., 2009). Finally, it is interesting to note that many, although not all (Poirier et al., 2007b; Daoud et al., 2008), mice models of human X-linked mental retardation syndromes due to gene mutation have profound deficits in object recognition memory (Alarcón et al., 2004; Vaillend et al., 2004; Ventura et al., 2004; Stearns et al., 2007; Arqué et al., 2008).

While several, although not all, of the above experiments have directly targeted the hippocampus, either by monitoring protein or gene activation after learning or via the use of hippocampalspecific gene deletion, a wide range of these molecular mechanisms were also shown to be engaged for recognition memory in other structures such as the perirhinal cortex (reviewed in Winters et al., 2008). Remarkably, all the molecular mechanisms thus far demonstrated to be critical for the consolidation of recognition memory are known mediators of synaptic long-term potentiation (LTP). This suggests that recognition memory, like many other forms of memory, engages synaptic plasticity. Clarke et al. (2010) recently reported evidence for this, showing that object exploration induces slow development of NMDA-dependent synaptic potentiation at the Schaffer collateral - CA1 pyramidal cell synapse in mice, a phenomenon occluded by prior induction of LTP, resulting in memory deficits.

A further point of interest concerns the potential role of hippocampal neurogenesis in recognition memory. It is now clearly established that new neurons are continuously generated in the dentate gyrus of the hippocampus and the past few years have witnesses accumulating evidence that young, newly generated dentate gyrus neurons play a significant role in several forms of hippocampaldependent memories (e.g., Ming and Song, 2005; Bruel-Jungerman et al., 2007; Deng et al., 2010 for reviews). Indirect evidence for a role of neurogenesis in object recognition memory first came from a study demonstrating a neurogenesis-dependent effect of environmental enrichment on the enhancement of long-term recognition memory (Bruel-Jungerman et al., 2005). Consistent with this, Jessberger et al. (2009) recently demonstrated that strong, but not partial, reduction of dentate gryus neurogenesis results in severe impairment in object recognition memory.

\section{RECONSOLIDATION OF OBJECT RECOGNITION MEMORY}

The first evidence that an object recognition memory can undergo reconsolidation after recall came from two experiments that examined what happens after a recall test in conditions of blocking MAPK/ERK activation or of inactivating zif268. In both conditions, the reconsolidation protocol consisted, one or several days after training, in a brief reactivation episode during which animals were placed back in the training environment with the same objects as those they have experienced during the training phase, followed 1 day later by a standard test during which one object was changed for a novel one. In the first experiment it was found that icv injection of a MEK inhibitor just prior to memory reactivation did not affect post-reactivation short-term memory but completely abolished the long-term post-reactivation memory that was observed in control rats (Kelly et al., 2003). These results suggested that memory for objects can be destabilized upon reactivation and that a process of reconsolidation, in this case dependent on MAPK/ERK activation, is required for the memory to be available for further long-term recall. At the same time, similar conclusions were reached from analyzing the effect of memory reactivation in zif 268 knockout mice. In this case, mice had first to be over-trained to override their consolidation deficit in the standard task so that they could form a long-term memory of objects. Then, when a brief reactivation trial was interposed between training and testing, long-term, but not short-term, post-reactivation memory was completely abolished (Bozon et al., 2003a). Further, the impairment after a brief reactivation was found to be specific to reactivation with the previously memorized objects in the relevant context; no impairment was observed when two novel objects were presented in the training context during the reactivation trial, or when the two previously experienced objects were presented in a novel and entirely different context. Moreover, the training context alone, which can often serve as a reminder cue, was not in this case an effective cue to trigger reactivation of the target object memory, suggesting that a zif268-dependent reconsolidation process acts on an configural memory associating attributes of the objects and of the context. Finally, we examined the temporal constraints on the requirement for zif268 in reconsolidation by varying the delay between training and reactivation, or between reactivation 
and test. Both recent ( 1 day) and relatively remote (4 days) memories were found to be subject to zif268-dependent reconsolidation after recall and there was no apparent spontaneous recovery of the memory for at least several days (Bozon et al., 2003a). The evidence that an object recognition memory can undergo reconsolidation after recall was recently confirmed using protein synthesis inhibition (Romero-Granados et al., 2010). In this study, systemic injection of anisomycin in mice just before or up to $4 \mathrm{~h}$ after memory reactivation using a similar test as above resulted in an impairment of postreactivation long-term memory, leaving short-term memory intact. Remote (21 days) object memory was also found to be susceptible to disruption by protein synthesis inhibition during recall. Object memory reconsolidation can also be disrupted by systemic injection of an NMDA receptor antagonist (Winters et al., 2009). In this case, however, reconsolidation of older or stronger memories became resistant to disruption by the NMDA antagonist unless a novel and salient contextual cue is present during reactivation.

In all, these results provide evidence that at least part of the same transcriptional and translational mechanisms involved in consolidation of object recognition memory are also engaged after recall and required for that memory to be available again for a further recall. Electrophysiological recordings of synaptic potentials at the Schaffer collateral-CA1 synapse during and after a retention test consisting in presenting a familiar with a novel object revealed a transient depression of synaptic potentials, possibly reflecting a destabilization mechanisms, followed by the slow development of synaptic potentiation similar to that observed after training (Clarke et al., 2010). This data suggest that at least this pathway undergo a similar form of synaptic change after both training and recall. The issue of whether or not both consolidation and reconsolidation of recognition memory engage the same brain circuits remains however debated. For example, protein synthesis inhibition in entorhinal cortex affects consolidation but not reconsolidation of object memory (Lima et al., 2009), while in the ventromedial prefrontal cortex, protein synthesis inhibition, as well as NMDA blockade or MAPK/ERK inhibition were shown to block both consolidation and reconsolidation of object memory (Akirav and Maroun, 2006; Maroun and Akirav, 2009). In our own MAPK/ERK studies, we found that learning about objects induces MAPK/ERK activation in the entorhinal cortex and dentate gyrus, while memory reactivation resulted in MAPK/ERK activation in entorhinal cortex and CA1, suggesting that cortico-hippocampal circuits engaged in consolidation and reconsolidation are at least in part distinct (Kelly et al., 2003). In a similar vein, BDNF and zif268 mRNA expression were reported in the entorhinal and prefrontal cortices after object sampling, while in this experiment reactivation 9 days after training induced zif268 in entorhinal and somatosensory cortices and upon reactivation 21 days after training, BDNF and not zif268 was expressed in hippocampus and entorhinal and somatosensory cortices (Romero-Granados et al., 2010). Thus, although certain circuits and mechanisms are common to both processes, there is not a strict match between the circuits engaged after training and recall of object memory and some of the signaling mechanisms seems to differ. It remain however difficult to construct a map of the mechanisms and circuits involved and several factors could account for some of the differences, such as details of the experimental procedure, strength of training, age of the memory, or the temporal dynamic of molecular changes.
Interestingly, recent evidence suggests reconsolidation of object memory can also be observed in humans. In an experiment where subjects were presented a first list of objects and then reminded or not before the presentation of a second list of objects, it was found that the reminder induced a misattribution of newly encoded objects (from list 2) into the reactivated list 1, suggesting that reactivation induced a reconsolidation process that served to modify the original object memory and update it with incorporation of objects belonging to list 2 (Hupbach et al., 2009).

\section{RECONSOLIDATION OF OBJECT-PLACE RECOGNITION MEMORY}

Experiments so far have demonstrated that object recognition memory can be destabilized after recall, requiring re-stabilization to re-enter a long-term store via a process that involves some but not all of the molecular mechanisms and brain circuits that are engaged in initial consolidation. Here we report novel experiments that extend these findings and demonstrate that objectplace recognition memory is also subject to reconsolidation after recall. The first experiment examined the potential role of the ribosomal S6 kinase Rsk2 in consolidation and reconsolidation of object-place recognition memory. The Rsk2 gene encodes a serine/threonine kinase that is activated by and acts downstream of MAPK/ERK via a dual function in CRE-mediated transcriptional regulation and in chromating remodeling by phosphorylating histone H3. In humans, Rsk2 gene mutations are responsible for a very handicapping X-linked form of syndromic mental retardation, the Coffin-Lowry syndrome (reviewed in Hanauer and Young, 2002; Pereira et al., 2010). In a previous experiment, we found that Rsk2 mutant mice have mild impairments in spatial working memory, delayed acquisition, and long-term memory deficits in spatial reference memory, but normal longterm object recognition memory (Poirier et al., 2007b). Thus, we examined whether Rsk2 might have a more prominent role in the more demanding spatial version of recognition memory, object-place recognition. Rsk2 and wild-type (WT) littermates were trained in a circular open-field covered with sawdust and containing three different objects constructed from assembling Lego ${ }^{\circledast}$ pieces. A cardboard cue was placed on the wall of the openfield to serve as a spatial landmark in addition to the multiple visual cues present in the environment. After habituation to the empty open-field for 2 days, Rsk 2 and WT mice were given three 5 -min trials of exploration of the objects with an inter-trial interval of 5 min. Retention was tested 2 days later during a single 5 -min trial by moving one of the objects to a new position. In the reconsolidation experiment, 1 day after training the mice were briefly re-exposed for $5 \mathrm{~min}$ to the three objects placed as in the training phase, and retention was tested 1 day later by moving one object to a new location. Analysis of the time spent exploring the displaced object revealed that Rsk2 deficiency did not cause any observable impairment in long-term object-place memory (Figure 1A). In the reconsolidation experiment, WT mice explored the displaced object significantly more than the two non-displaced objects (Figure 1B), demonstrating a similar recognition performance to that when no reactivation was interposed. Surprisingly, however, while post-reactivation shortterm memory was intact in Rsk 2 deficient mice, post-reactivation 
long-term memory was completely abolished (Figure 1B). These findings demonstrate that object-place memory is subject to a Rsk2-dependent reconsolidation process following memory reactivation and provide an example of a divergence between mechanisms of consolidation and reconsolidation of recognition memory by showing that the signaling molecule Rsk 2 is at least more prominently implicated in object-place memory reconsolidation than in object-place memory consolidation.
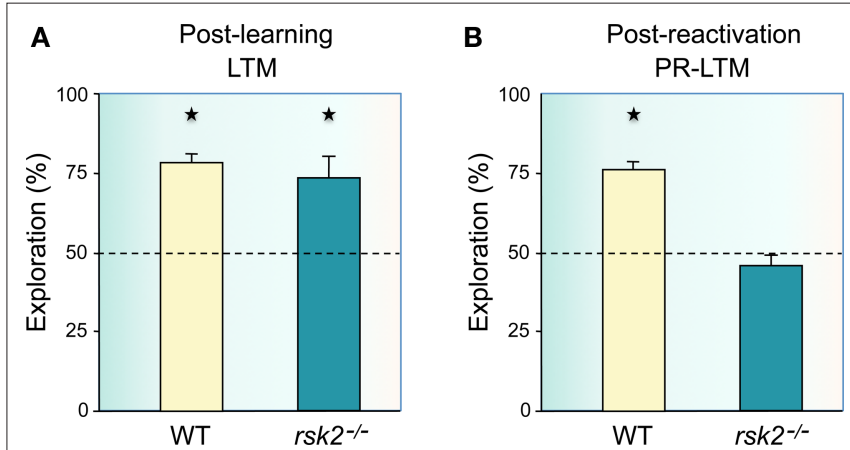

FIGURE 1 | Reconsolidation, but not consolidation of spatial, objectplace recognition memory is impaired in Rsk2 mutant mice. (A) Rsk2 mutant mice showed no deficit in long-term spatial recognition memory (LTM) over $48 \mathrm{~h}$ as they showed preferential exploration of the displaced object ( $n=13 ; t=3.53 ; p=0.0041)$ as did wild-type (WT) mice $(n=13 ; t=9.83$; $p=0.0001$ ), with no significant difference in the amount of time spent exploring the displaced object between WT and mutant mice $\left(F_{1,24}=0.416\right.$; $p=0.525)$. (B) In contrast, 24 h after reactivation of the memory, Rsk2 mutant mice showed a deficit as they displayed no preference for the displaced object ( $t=1.43 ; p=0.17)$ as opposed to the WT mice $(t=14.61 ; p=0.0001)$; and the level of exploration of the displaced object was significantly greater in WT mice compared with mutant mice $\left(F_{124}=70.753 ; p=0.0001\right)$. Ordinates: percent time spent exploring the displaced object over the mean of the time spent exploring the two non-displaced objects.
In the second experiment to examine whether object-place recognition memory can become destabilized after recall and requires a process of reconsolidation to maintain the memory for further use, we tested zif268 mutant mice, which have been previously shown to be impaired in object memory reconsolidation (Bozon et al., 2003a). As in the Bozon and colleagues' experiment, we first tested whether zif268 mutant mice could form a long-term object-place memory if given additional exposures to the objects in a distributed training paradigm, a precondition to examine the potential role of zif268 in reconsolidation. WT and zif268 mutant mice were given four blocks of two 5-min trials of exploration of two different objects with a within-block intertrial interval of 5 min and a 90-min interval between blocks, and retention was measured 2 days later by moving one of the objects to a novel location. Both WT and zif268 mutant mice showed preferential exploration of the displaced object (Figure 2A), thus demonstrating the mice can form a long-term object-place memory in conditions of extended and distributed training. We were thus able to explore the effect of a brief reactivation trial (a single 5-min session with the objects in the same locations as during training), interposed at a 1-day interval between training and retention. When post-reactivation short-term memory was tested, both WT and zif268 mutant mice showed preferential exploration of the displaced object (Figure 2B, PR-STM). One day after reactivation, WT mice also explored significantly more the displaced object (Figure 2B, PR-LTM), demonstrating a similar recognition performance to that when no reactivation was interposed. In contrast, zif268 mutant mice showed equal exploration of the two objects (Figure 2B). These findings demonstrate that a consolidated and stable object-place recognition memory can again become labile after brief reactivation and zif 268 mutant mice cannot in this case reconsolidate the object-place memory. Thus a zif268-dependent reconsolidation process is similarly required after an object memory or an object-place memory is recalled.

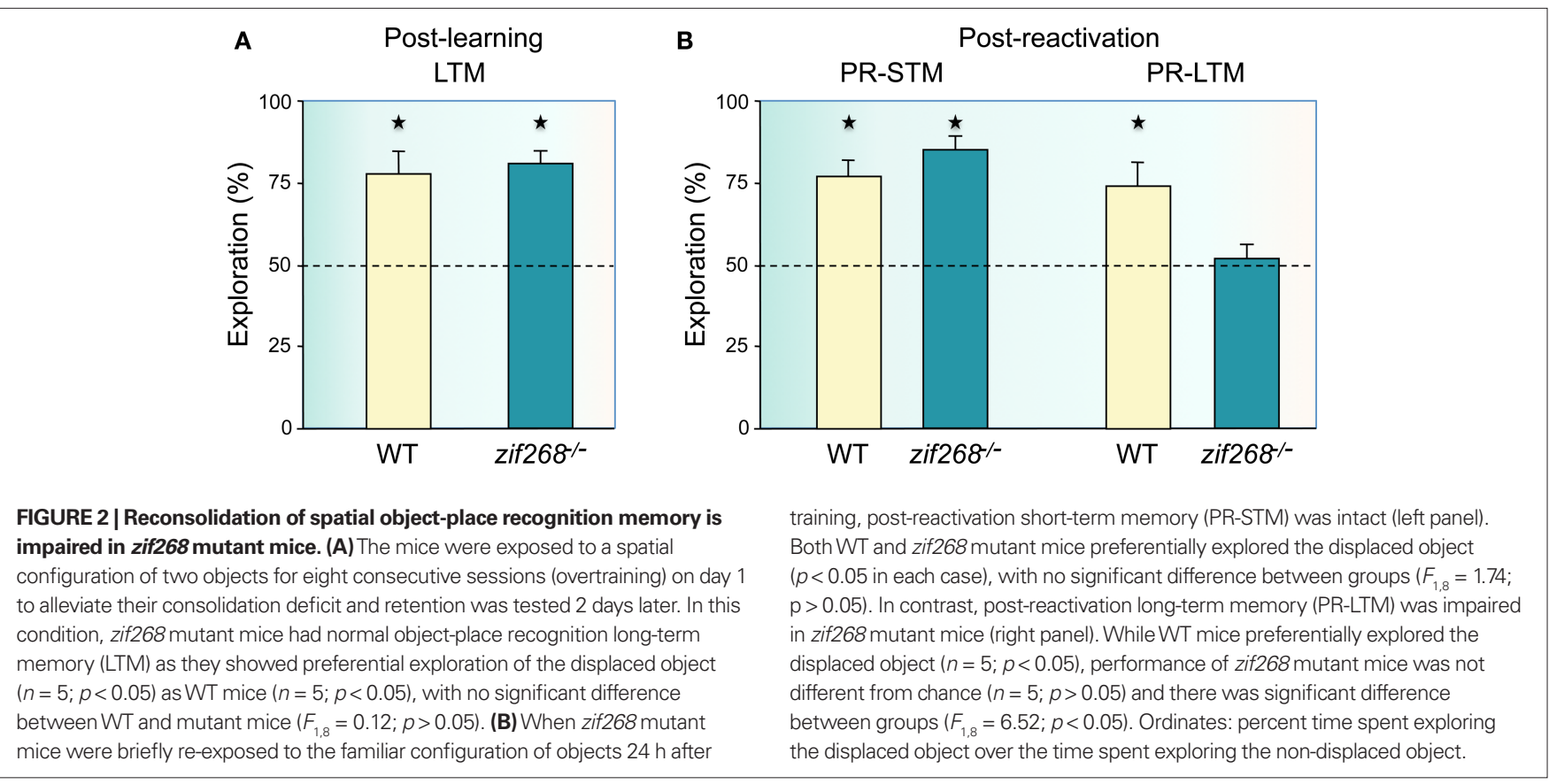




\section{CONCLUDING COMMENTS}

The demonstration that many forms of memory can be subjected to reconsolidation after recall has opened a new era in memory research, highlighting its dynamic and reconstructive nature. In a little over a decade, a considerable number of studies have been conducted on different aspects of reconsolidation in an attempt to understand the conditions under which this process occurs, what its function is and what the underlying mechanisms are. Whereas a large number of these studies have used fear-associated or negatively reinforced memories, relatively few to date have addressed the issue of what happens after recall of forms of recognition memory that are based on incidental encoding with no explicit reinforcement. Yet, recognition memory is a necessary component of nearly all forms of memories, be they pathological or normal. As reviewed in the preceding sections, it is now clearly established that both object and object-place recognition memory can become destabilized after recall and would then require reconsolidation to remain available for further recall. The evidence comes from several sources demonstrating that when signaling molecules or genes implicated in the type of synaptic plasticity believed to underlie memory stabilization are interfered with at the time of memory reactivation, the memory of a familiar object or of a familiar object-place association is lost. The findings thus extend to recognition memory the theoretical account put forth by Lewis (1979), suggesting that memory of past encountered objects can return to a labile state and become vulnerable to disruptive factors after recall before eventually being re-stabilized as a result of their reactivation.

What are the conditions under which recognition memories undergo reconsolidation after recall? Clearly, there is still a lack of behavioral experiments on recognition memory to come to a detailed account on the issue. Yet certain basic features can be delineated. There is some evidence for example to suggest that effective post-retrieval "amnestic" conditions affect reconsolidation of both recent and relatively remote recognition memory, although this type of memory is usually far less enduring than negatively reinforced memories, which makes it impractical to test over a long time range. There is also some evidence to suggest that the apparent amnesia after reconsolidation blockade is not reversible. It is not known however whether this is a complete loss of all items of information that the episode includes or not. Cueing experiments after reconsolidation blockade could provide valuable information as to whether or not some elements of the memory are spared, and could help attack the general issue of whether the deficit in performance reflects impaired re-stabilization of the memory or rather affects retrieval processes, which is not better resolved in the case of recognition memory than it is for other forms of memories.

There is also indication that to be susceptible to disruption, reactivation of object recognition memory may have to be prompted by presenting the target memory (the objects) in the relevant context. A peculiarity of the experimental design used for reactivating recognition memory is that the reactivation session consists in presenting the same, and whole event that was present during the encoding phase, whereas in many other types of learning partial information alone (e.g., the conditioned stimulus, the context associated with a reinforcer) can reactivate the memory without the need for giving the reinforcer. This may be due to the importance of context in many paradigms of recognition memory in the absence of explicit reinforcement, apart for the natural, ecologically relevant tendency of rodents to attend to, and explore novel items in their environment. It does not preclude, however, the possibility that a past object memory can be reactivated in conditions of only partial similarity between initial training and reactivation, as shown in human studies (Hupbach et al., 2007). Inherent in recognition memory tests is the fact that during reactivation, as well as during testing with a novel object or a displaced object, memories of the objects or their location that are first encountered must be reactivated as a means of determining whether they are familiar or novel. Evidence in humans and rodents suggest that the hippocampus plays a key role in novelty detection by acting as a comparator between past and current overlapping, or similar events (Kumaran and Maguire, 2007; Lever et al., 2010). Computational models suggest this is achieved by a match-mismatch comparator system to generate orthogonal memory representations via a pattern separation function, which may occur in the object recognition test when an object or location of an object is changed. In order to make comparisons between familiar and novel events, the comparator system also requires a mechanism whereby stored representations can be fully recalled in response to a minimal amount of input cue via pattern completion (Lee and Kesner, 2004; Rolls, 2010). At present, given the procedure used for reconsolidation in rodents, it seems that a match condition is required for post-retrieval "amnestic" treatments to destabilize the memory, however, further experiments will be needed to explore whether a non-match condition either does not destabilize the past memory, rendering reconsolidation unnecessary, or does destabilize it in a manner that is less sensitive to treatments. Consistent with the latter possibility, in a previous experiment investigating CA1 place cell representations in zif268 knockout mice (Renaudineau et al., 2009), we found that the mice have deficits in stabilizing long-term place cell representations of a novel environment containing an object, which is consistent with their impaired long-term spatial memory, and also that an established place cell representation of a well-experienced environment (corresponding to an overtraining procedure) can be destabilized by placing the mice in a slightly different environment. Thus, the formation of the new representation interfered with the ability to later reactivate the representation of a familiar environment. This did not result in a complete loss however, but lead to rotational remapping by the place cell system associated with rate change, indicating an incorrect orientation of the place cell map corresponding to the familiar representation, rather than creation of a totally new representation. These findings suggest the memory of the familiar environment was destabilized, although further successive exposures to the familiar and novel environments progressively suppressed this destabilizing effect of the now less novel environment (Renaudineau et al., 2009). One possible explanation is that formation of the new representation triggers a form of reconsolidation that renders the previously formed representation labile and vulnerable to interference; the absence of zif268 in this case preventing proper re-stabilization of the former representation. Relevant to this, a recent fMRI study in normal subjects submitted to object lists learning provided evidence for reactivation of a previously encoded memory during the encoding of a new, partially overlapping list and suggested the hippocampus takes part in reactivating the older memory when a new memory is formed, 
limiting the forgetting of the older memory (Kuhl et al., 2010). Within this framework, it is possible that the absence of zif268 in the hippocampus was deleterious to a process of reconsolidation of the reactivated older representation.

The issue of whether or not consolidation and reconsolidation engage some of the same biochemical/molecular mechanisms has been raised since the start of research on reconsolidation, in particular because most "amnestic" treatments used in reconsolidation studies target molecular mechanisms known to be involved in consolidation of new memories. From the studies reviewed above, two conclusions can de drawn. First, there is evidence that consolidation and reconsolidation of recognition memory engage common mechanisms, including NMDA receptor activation, MAPK/ERK phosphorylation, zif268 transcriptional regulation, and protein synthesis. There are also similarities in the mechanisms involved in reconsolidation of both object and object-place memories. However, there are also arguments to suggest that certain mechanisms may be more specific to one or the other process, as exemplified by the prominent requirement of Rsk2, a MAPK/ERK substrate, in reconsolidation but not consolidation of object-place recognition memory. Second, some of the studies directed at exploring the cellular and molecular mechanisms of consolidation and reconsolidation of recognition memory that were based on detecting activation of specific cell-signaling cascades in different neuronal populations or using structure specific genetic deletion further emphasizes the implication of the hippocampus in both object and object-place recognition memory. However, several examples indicate that the brain structures within which some of these molecular mechanisms take place, including within cortico-hippocampal circuits, do not completely overlap between consolidation and reconsolidation of recognition memory. Admittedly, the available data on

\section{REFERENCES}

Akirav, I., and Maroun, M. (2006). Ventromedial prefrontal cortex is obligatory for consolidation and reconsolidation of object recognition memory. Cereb. Cortex 16, 1759-1765.

Alarcón, J. M., Malleret, G., Touzani, K., Vronskaya, S., Ishii, S., Kandel, E. R., and Barco, A. (2004). Chromatin acetylation, memory, and LTP are impaired in $\mathrm{CBP}+/-$ mice: a model for the cognitive deficit in RubinsteinTaybi syndrome and its amelioration. Neuron 42, 947-959.

Alberini, C. M. (2005). Mechanisms of memory stabilization: are consolidation and reconsolidation similar or distinct processes? Trends Neurosci. 28, 51-56.

Arqué, G., Fotaki, V., Fernández, D., Martínez de Lagrán, M., Arbonés, M. L., and Dierssen, M. (2008). Impaired spatial learning strategies and novel object recognition in mice haploinsufficient for the dual specificity tyrosineregulated kinase-1A (Dyrk1A). PLoS ONE 3, e2575. doi: 10.1371/journal. pone. 0002575 .
Artinian, J., Jaeger, X. D., Fellini, L. Blanquat, P. d. S., and Roullet, P. (2007). Reactivation with a simple exposure to the experimental environment is sufficient to induce reconsolidation requiring protein synthesis in the hippocampal CA3 region in mice. Hippocampus 17, 181-191.

Balderas, I., Rodriguez-Ortiz, C. J., Salgado-Tonda, P., Chavez-Hurtado, J., McGaugh, J. L., and BermudezRattoni, F. (2008). The consolidation of object and context recognition memory involve different regions of the temporal lobe. Learn. Mem. 15, 618-624.

Barrett, R., and Wood, M. A. (2008). Beyond transcription factors: the role of chromatin modifying enzymes in regulating transcription required for memory. Learn. Mem. 15, 460-467.

Bozon, B., Davis, S., and Laroche, S. (2002). Regulated transcription of the immediate early gene Zif268: mechanisms and gene dosage-dependent function in synaptic plasticity and $12,570-577$. memory formation. Hippocampus

the mechanisms and brain circuits engaged in consolidation and reconsolidation of recognition memory remains too limited to draw firm conclusions. In addition, at this point these data do not tell us what specific function(s) the hippocampus may implement in recognition memory consolidation or reconsolidation, nor do they tell us whether reconsolidation strengthens or updates the memory. Future research to achieve a more complete understanding of the cellular and molecular mechanisms and of the brain circuits engaged in reconsolidation of recognition memory will serve to further our knowledge of the function of these circuits in reconsolidation and of the function of reconsolidation in the makings of memories. Determining how neuronal firing patterns in different brain regions encode independent aspects of recognition memory, such as individual items, ensembles of items, spatial, and non-spatial context, and the temporal order in which events occur, could be one possible route into gaining a better understanding of the dynamics of the processing, recall, and the circumstances in which memory for incidental learning may require a process of reconsolidation.

\section{ACKNOWLEDGMENTS}

Research presented in this article was funded by CNRS, Université Paris-Sud, Université Aix-Marseille and by an ACI grant (NIC0027) from the French Ministry of Research to Sabrina Davis and Bruno Poucet and an ANR (ANR-05-NEUR-005-01) grant to Serge Laroche. Roseline Poirier was supported by a fellowship from the Neuropôle de Recherche Francilien (NeRF). We thank André Hanauer for generously providing Rsk2 mutant mice. Zif268 mutant mice were bred in Orsay from breeders generously provided by Patrick Charnay and Piotr Topilko. We are grateful to Pascale Veyrac, Nathalie Devignes and Solange Pannetier for providing animal care and for rearing and genotyping the mice.

Bozon, B., Davis, S., and Laroche, S. (2003a).A requirement for the immediate early gene zif268 in reconsolidation of recognition memory after retrieval. Neuron 40, 695-701.

Bozon, B., Kelly, A., Josselyn, S. A., Silva, A. J., Davis, S., and Laroche, S. (2003b) MAPK, CREB and zif268 are all required for the consolidation of recognition memory. Phil. Trans. R. Soc B 358, 805-814.

Broadbent, N. J., Gaskin, S., Squire, L. R. and Clark, R. E. (2009). Object recognition memory and the rodent hippocampus. Learn. Mem. 17, 5-11.

Broadbent, N. J., Squire, L. R., and Clark, R. E. (2004). Spatial memory, recognition memory, and the hippocampus. Proc. Natl. Acad. Sci. U.S.A. 101, 14515-14520.

Brown, M. W., and Aggleton, J. P. (2001) Recognition memory: what are the roles of the perirhinal cortex and hippocampus? Nat. Rev. Neurosci. 2, 51-61.

Bruel-Jungerman, E., Laroche, S., and Rampon, C. (2005). New neurons in the dentate gyrus are involved in the expression of enhanced long-term memory following environmental enrichment. Eur. J. Neurosci. 21, 513-521.

Bruel-Jungerman, E., Rampon, C., and Laroche, S. (2007). Adult hippocampal neurogenesis, synaptic plasticity and memory: facts and hypotheses. Rev. Neurosci. 18, 93-114.

Clark, R. E., Zola, S. M., and Squire, L. R. (2000). Impaired recognition memory in rats after damage to the hippocampus. J. Neurosci. 20, 8853-8860.

Clarke, J. R., Cammarota, M., Gruart, A. Izquierdo, I., and Delgado-García, J. M. (2010). Plastic modifications induced by object recognition memory processing. Proc. Natl. Acad. Sci. U.S.A. 107, 2652-2657.

Coleshill, S. G., Binnie, C. D., Morris, R. G., Alarcón, G., van Emde Boas, W., Velis, D. N., Simmons, A., Polkey, C. E., van Veelen, C. W. M., and van Rijen, P. C. (2004). Material-specific recognition memory deficits elicited by unilateral hippocampal electrical stimulation. J. Neurosci. 24, 1612-1616. 
Daoud,F.,Candelario-Martínez,A.,Billard, J.M.,Avital,A., Khelfaoui,M.,Rozenvald, Y., Guegan, M., Mornet, D., Jaillard, D., Nudel, U., Chelly, J., Martínez-Rojas, D., Laroche, S., Yaffe, D., and Vaillend, C. (2008). Role of mental retardationassociated dystrophin-gene product Dp71 in excitatory synapse organization, synaptic plasticity and behavioral functions. PLoS ONE 4, e6574. doi: 10.1371/journal.pone.0006574.

Davis, S., and Laroche, S. (2006). Mitogenactivated protein kinase/extracellular regulated kinase signalling and memory stabilization: a review. Genes Brain Behav. 5, 61-72.

Davis,S., Vanhoutte,P.,Pages, C., Caboche,J., and Laroche,S. (2000). The MAPK/ERK cascade targets both elk-1 and cAMP response element-binding protein to controllong-term potentiation-dependent gene expression in the dentate gyrus in vivo. J. Neurosci. 20, 4563-4572.

Debiec, J., Doyère, V., Nader, K., and Ledoux, J. E. (2006). Directly reactivated, but not indirectly reactivated, memories undergo reconsolidation in the amygdala. Proc. Natl. Acad. Sci. U.S.A. 103, 3428-3433.

Debiec, J., LeDoux, J. E., and Nader, K. (2002). Cellular and systems reconsolidation in the hippocampus. Neuron 36, 527-538.

de Lima, M. N., Luft, T., Roesler, R., and Schröder, N. (2006). Temporary inactivation reveals an essential role of the dorsal hippocampus in consolidation of object recognition memory. Neurosci. Lett. 405, 142-146.

Deng, W., Aimone, J. B., and Gage, F. H. (2010). New neurons and new memories: how does adult hippocampal neurogenesis affect learning and memory? Nat. Rev. Neurosci. 11, 339-350.

Dere, E., Huston, J. P., and De Souza Silva, M. A. (2007). The pharmacology, neuroanatomy and neurogenetics of one-trial object recognition in rodents. Neurosci. Biobehav. Rev. 31, 673-704.

Dudai, Y., and Eisenberg, M. (2004). Rites of passage of the engram: reconsolidation and the lingering consolidation hypothesis. Neuron 44, 93-100.

Eichenbaum, H., Yonelinas, A. R., and Ranganath, C. (2007). The medial temporal lobe and recognition memory. Annu. Rev. Neurosci. 30, 123-152.

Ennaceur, A., and Delacour, J. (1988). A new one-trial test for neurobiological studies of memory in rats. 1: behavioral data. Behav. Brain. Res. 31, 47-59.

Gordon, W. C., and Spear, N. E. (1973). Effect of reactivation of a previously acquired memory on the interaction between memories in the rat. J. Exp. Psychol. 99, 349-355.

Hammond, R. S., Tull, L.E., and Stackman, R.W. (2004). On the delay-dependent involvement of the hippocampus in object recognition memory. Neurobiol. Learn. Mem. 82, 26-34.

Hanauer, A., and Young, I. D. (2002) Coffin-Lowry syndrome: clinical and molecular features. J. Med. Genet. 39, 705-713.

Hardt, O., Einarsson, E. O., and Nader, K. (2010). A bridge over troubled water: reconsolidation as a link between cognitive and neuroscientific memory research traditions. Annu. Rev. Psychol. 61, 141-167.

Horwood, J. M., Dufour, F., Laroche, S., and Davis, S. (2006). Signalling mechanisms mediated by the phosphoinositide 3-kinase/Akt cascade in synaptic plasticity and memory in the rat. Eur. J. Neurosci. 23, 3375-3384.

Hupbach, A., Gomez, R., Hardt, O., and Nadel, L. (2007). Reconsolidation of episodic memories: a subtle reminder triggers integration of new information. Learn. Mem. 14, 47-53.

Hupbach, A., Gomez, R., and Nadel, L. (2009). Episodic memory reconsolidation: updating or source confusion? Memory 17, 502-510.

Jenkins, T.A.,Amin, E.,Pearce,J.M., Brown, M. W., and Aggleton, J. P. (2004). Novel spatial arrangements of familiar visual stimuli promote activity in the rat hippocampal formation but not the parahippocampal cortices: ac-fos expression study. Neuroscience 124, 43-52.

Jessberger, S., Clark, R. E., Broadbent, N. J., Clemenson, G. D.Jr.,Consiglio,A.,Lie,D. C., Squire, L. R., and Gage, F. H. (2009). Dentate gyrus-specific knockdown of adult neurogenesis impairs spatial and object recognition memory in adult rats. Learn. Mem. 16, 147-154.

Jones, M. W., Errington, M. L., French, P. J., Fine, A., Bliss, T. V. P., Garel, S. Charnay, P., Bozon, B., Laroche, S. and Davis, S. (2001). A requirement for the immediate early gene Zif268 in the expression of late LTP and the consolidation of long-term memories. Nat. Neurosci. 4, 289-296.

Kelly, A., Laroche, S., and Davis, S. (2003) Activation of mitogen-activated protein kinase/extracellular signalregulated kinase in hippocampal circuitry is required for consolidation and reconsolidation of recognition memory. J. Neurosci. 12, 5354-5360.

Kida, S., Josselyn, S. A., Peña de Ortiz, S. Kogan, J.H., Chevere, I., Masushige, S., and Silva, A. J. (2002).CREB required for the stability of new and reactivated fear memories. Nat. Neurosii. 5, 348-355.

Koshibu, K., Gräff, J., Beullens, M. Heitz, F. D., Berchtold, D., Russig, H., Farinelli, M., Bollen, M., and Mansuy, I. M. (2009). Protein phosphatase 1 regulates the histone code for long-term memory. J. Neurosci. 29 , 13079-13089.
Kuhl, B. A., Shah, A. T., DuBrow S, and Wagner, A. D. (2010). Resistance to forgetting associated with hippocampus-mediated reactivation during new learning. Nat. Neurosci. 13, 501-506.

Kumaran, D., and Maguire, E. A. (2007) Match mismatch processes underlie human hippocampal responses to associative novelty. J. Neurosci. 27 8517-8524.

Languille, S., Davis, S., Richer, P., Alcacer C., Laroche, S., and Hars, B. (2009). Extracellular signal-regulated kinase activation is required for consolidation and reconsolidation of memory at an early stage of ontogenesis. Eur. J. Neurosci. 30, 1923-1930.

Lee, I., and Kesner, R. P. (2004). Encoding versus retrieval of spatial memory: double dissociation between the dentate gyrus and the perforant path inputs into CA3 in the dorsal hippocampus. Hippocampus 14, 66-76.

Lee, J. L. (2009). Reconsolidation: maintaining memory relevance. Trends Neurosci. 32, 413-420.

Lee, J. L., Everitt, B. J., and Thomas, K. L. (2004).Independent cellular processes for hippocampalmemoryconsolidation and reconsolidation. Science 304, 839-843.

Lever, C., Burton, S., Jeewajee, A., Wills, T. J Cacucci, F., Burgess, N., and O'Keefe, J. (2010). Environmental novelty elicits a later theta phase of firing in $\mathrm{CAl}$ but not subiculum. Hippocampus 20, 229-234.

Lewis, D. J. (1979). Psychobiology of active and inactive memory. Psychol. Bull. 86 , 1054-1083.

Li, L., Yun, S. H., Keblesh, J., Trommer B. L., Xiong, H., Radulovic, J., and Tourtellotte, W. G. (2007). Egr3, a synaptic activity regulated transcription factor that is essential for learning and memory. Mol. Cell. Neurosci. $35,76-88$.

Lima, R. H., Rossato, J. I., Furini, C. R., Bevilaqua, L. R., Izquierdo, I., and Cammarota, M. (2009). Infusion of protein synthesis inhibitors in the entorhinal cortex blocks consolidation but not reconsolidation of object recognition memory. Neurobiol. Learn Mem. 91, 466-472.

Litvin, O. O., and Anokhin, K. V. (2000). Mechanisms of memory reorganization during retrieval of acquired behavioral experience in chicks: the effects of protein synthesis inhibition in the brain. Neurosci. Behav. Physiol. 30, 671-678.

Malleret, G., Haditsch, U., Genoux, D., Jones, M.W., Bliss, T.V.P., Vanhoose, A. M., Weitlauf, C., Kandel, E. R., Winder, D. G., and Mansuy, I. M. (2001) Inducible and reversible enhancement of learning, memory, and long-term potentiation by genetic inhibition of calcineurin. Cell 104, 675-686.

Manns, J. R., and Eichenbaum, H. (2009). A cognitive map for object memory in the hippocampus. Learn. Mem. 16, 616-624.

Maroun, M., and Akirav, I. (2009). Differential involvement of dopamine D1 receptor and MEK signaling pathway in the ventromedial prefrontal cortex in consolidation and reconsolidation of recognition memory. Learn. Mem. 16, 243-247.

Milekic, M.H., and Alberini, C. M. (2002). Temporally graded requirement for protein synthesis following memory reactivation. Neuron 36, 521-525.

Miller, S., Yasuda, M., Coats, J. K., Jones, Y., Martone, M. E., and Mayford, M. (2002). Disruption of dendritic translation of CaMKIIalpha impairs stabilization of synaptic plasticity and memory consolidation. Neuron 36, 507-519.

Ming, G. L., and Song, H. (2005). Adult neurogenesis in the mammalian central nervous system. Annu. Rev. Neurosci. 28, 223-250.

Misanin, J. R., Miller, R. R., and Lewis, D.J. (1968). Retrograde amnesia produced by electroconvulsive shock after reactivation of a consolidated memory trace. Science 160, 554-555.

Morris, R. G. M., Inglis, J., Ainge, J. A., Olverman, H. J., Tulloch, J., Dudai, Y., and Kelly, P.A. (2006). Memory reconsolidation: sensitivity of spatial memory to inhibition of protein synthesis in dorsal hippocampus during encoding and retrieval. Neuron 50, 479-489.

Mumby, D. G. (2001). Perspectives on object-recognition memory following hippocampal damage: lessons from studies in rats. Behav. Brain Res. 127, 159-181.

Myskiw, J. C., Rossato, J. I., Bevilaqua, L. R., Medina, J. H., Izquierdo, I., and Cammarota, M. (2008). On the participation of $\mathrm{mTOR}$ in recognition memory. Neurobiol. Learn. Mem. 89, 338-351.

Nader, K., and Einarsson, E. O. (2010). Memory reconsolidation: an update. Ann. N. Y. Acad. Sci. 1191, 27-41.

Nader, K., and Hardt, O. (2009). A single standard for memory: the case for reconsolidation. Nat. Rev. Neurosci. $10,224-234$.

Nader, K., Schafe, G. E., and Le Doux, J. E. (2000). Fear memories require protein synthesis in the amygdala for reconsolidation after retrieval. Nature 406, 722-726.

Nemanic, S., Alvarado, M. C., and Bachevalier, J. (2004). The hippocampal/parahippocampal regions and recognition memory: insights from visual paired comparison versus object-delayed nonmatching in monkeys. J. Neurosci. 24, 2013-2026.

Pereira, P. M., Schneider, A., Pannetier, S., Heron, D., and Hanauer, A. (2010). Coffin-Lowry syndrome. Eur. J. Hum Genet. 18, 627-633. 
Pittenger, C., Huang, Y. Y., Paletzki, R. F., Bourtchouladze, R., Scanlin, H., Vronskaya, S., and Kandel, E. R. (2002). Reversible inhibition of CREB/ ATF transcription factors in region CAl of the dorsal hippocampus disrupts hippocampus-dependent spatial memory. Neuron 34, 447-462.

Plath, N., Ohana, O., Dammermann, B., Errington, M. L., Schmitz, D., Gross, C., Mao, X., Engelsberg, A., Mahlke, C., Welzl, H., Kobalz, U., Stawrakakis, A., Fernandez, E., Waltereit, R., BickSander, A., Therstappen, E., Cooke, S. F., Blanquet, V., Wurst, W., Salmen, B., Bösl, M. R., Lipp, H. P., Grant, S. G., Bliss, T. V. P., Wolfer, D. P., and Kuhl, D. (2006). Arc/Arg3.1 is essential for the consolidation of synaptic plasticity and memories. Neuron 52, 437-444.

Poirier, R., Cheval, H., Mailhes, C., Charnay, P., Davis, S., and Laroche, S. (2007a). Paradoxal role of an Egr transcription factor family member, Egr2/Krox20, in learning and memory. Front. Behav. Neurosci. 1:6. doi: 10.3389/neuro.08/006.2007.

Poirier, R., Jacquot, S., Vaillend, C., Soutthiphong, A. A., Libbey, M., Davis, S., Laroche, S., Hanauer, A., Welzl, H., Lipp, H. P., and Wolfer, D. P. (2007b). Deletion of the Coffin-Lowry syndrome gene Rsk2 in mice is associated with impaired spatial learning and reduced control of exploratory behavior. Behav. Genet. 37, 31-50.

Poirier, R., Cheval, H., Mailhes, C., Garel, S., Charnay, P., Davis, S., and Laroche, S. (2008). Distinct functions of egr gene family members in cognitive processes. Front. Neurosci. 2, 47-55. doi: 10.3389/neuro.01.002.2008.

Power, A. E., Berlau, D. J., McGaugh, J. L., and Steward, O. (2006). Anisomycin infused into the hippocampus fails to block "reconsolidation" but impairs extinction: the role of re-exposure duration. Learn. Mem. 13, 27-34.

Przybyslawski, J., and Sara, S. J. (1997). Reconsolidation of memory after its reactivation. Behav. Brain Res. 84, 241-246.

Renaudineau, S., Poucet, B., Laroche, S., Davis, S., and Save, E. (2009). Impaired long-term stability of CA1 place cell representation in mice lacking the transcription factor zif268/egr 1. Proc. Natl. Acad. Sci. U.S.A. 106, 11771-11775.

Robinson, M. J., and Franklin, K. B. (2010). Reconsolidation of a morphine place preference: impact of the strength and age of memory on disruption by propranolol and midazolam. Behav. Brain Res. [Epub ahead of print]. PMID: 20457186.

Rolls, E. T. (2010).A computational theory of episodic memory formation in the hippocampus. Behav. Brain Res. [Epub ahead of print]. PMID: 20307583.
Romero-Granados, R., Fontán-Lozano,A., Delgado-García, J. M., and Carrión, A. M. (2010). From learning to forgetting: behavioral, circuitry, and molecular properties define the different functional states of the recognition memory trace. Hippocampus 20, 584-595.

Rose, J. K., and Rankin, C. H. (2006). Blocking memory reconsolidation reverses memory-associated changes in glutamate receptor expression. $J$. Neurosci. 26, 11582-11587.

Rossato, J. I., Bevilaqua, L. R., Myskiw, J. C., Medina, J. H., Izquierdo, I., and Cammarota, M. (2007). On the role of hippocampal protein synthesis in the consolidation and reconsolidation of object recognition memory. Learn. Mem. 14, 36-46.

Rugg, M. D., and Curran, T. (2007). Eventrelated potentials and recognition memory. Trends Cogn. Sci. 11, 251-257.

Sangha, S., Scheibenstock, A., and Lukowiak, K. (2003). Reconsolidation of a long-term memory in Lymnaea requires new protein and RNA synthesis and the soma of right pedal dorsal 1. J. Neurosci. 23, 8034-8040.

Sauvage, M. M., Fortin, N.J., Owens, C. B., Yonelinas, A. P., and Eichenbaum, H. (2008). Recognition memory: opposite effects of hippocampal damage on recollection and familiarity. Nat. Neurosci. 11, 16-18.

Schiller, D., Monfils, M. H., Raio, C. M., Johnson, D.C., Ledoux, J.E., and Phelps, E.A. (2010).Preventing the return of fear in humans using reconsolidation update mechanisms. Nature 463, 49-53.

Schwabe, L., and Wolf, O. T. (2009). New episodic learning interferes with the reconsolidation of autobiographical memories. PLoS ONE 4, 7519. doi: 10.1371/journal.pone.0007519.

Skinner, E. I., and Fernandes, M.A. (2007). Neural correlates of recollection and familiarity: a review of neuroimaging and patient data. Neuropsychologia 45 , 2163-2179.

Soulé, J., Penke, Z., Kanhema, T., Alme, M. N., Laroche, S., and Bramham, C. R. (2008). Object-place recognition learning triggers rapid induction of plasticity-related immediate early genes and synaptic proteins in the rat dentate gyrus. Neural Plast. 2008, 269097.

Squire, L. R., Wixted, J. T., and Clark, R.E. (2007). Recognition memory and the medial temporal lobe: a new perspective. Nat. Rev. Neurosci. 8, 872-883.

Stearns, N. A., Schaevitz, L. R., Bowling, H., Nag, N., Berger, U. V., and BergerSweeney, J. (2007). Behavioral and anatomical abnormalities in Mecp2 mutant mice: a model for Rett syndrome. Neuroscience 146, 907-921.

Stefanko, D. P., Barrett, R. M., Ly, A. R., Reolon, G. K., and Wood, M.A. (2009). Modulation of long-term memory for object recognition via HDAC inhibition. Proc. Natl. Acad. Sci. U.S.A 106, 9447-9452.

Stollhoff, N., Menzel, R., and Eisenhardt, D. (2005). Spontaneous recovery from extinction depends on the reconsolidation of the acquisition memory in an appetitive learning paradigm in the honeybee (Apis mellifera). J. Neurosci. 25, 4485-4492.

Suzuki, A., Josselyn, S. A., Frankland, P.W. Masushige, S., Silva, A. J., and Kida, S. (2004). Memory reconsolidation and extinction have distinct temporal and biochemical signatures. J. Neurosci. 24 , 4787-4795.

Sweatt,J.D.(2001).Theneuronal MAPkinase cascade: a biochemical signal integration system subserving synaptic plasticity and memory. J. Neurochem. 76, 1-10.

Taubenfeld, S. M., Milekic, M. H., Monti, B., and Alberini, C. M. (2001). The consolidation of new but not reactivated memory requires hippocampal C/EBPbeta. Nat. Neurosci. 4 813-818.

Taylor, J. R., Olausson, P., Quinn, J. J., and Torregrossa, M. M. (2009). Targeting extinction and reconsolidation mechanisms to combat the impact of drug cues on addiction. Neuropharmacology 56, 186-195.

Tronson, N. C., and Taylor, J. R. (2007). Molecular mechanisms of memory reconsolidation. Nat. Rev. Neurosci. 8, 262-275.

Tse, D., Langston, R. F., Kakeyama, M., Bethus, I., Spooner, P. A., Wood, E. R., Witter, M. P., and Morris, R. G. M. (2007). Schemas and memory consolidation. Science 316, 76-82.

Tulving, E. (2002). Episodic memory: from mind to brain. Annu. Rev Psychol. 53, 1-25.

Vaillend, C., Billard, J. M., and Laroche, S. (2004). Impaired long-term spatial and recognition memory and enhanced CA1 hippocampal LTP in the dystrophin-deficient $\operatorname{Dmd}(\mathrm{mdx})$ mouse. Neurobiol. Dis. 17, 10-20.

Ventura, R., Pascucci, T., Catania, M. V., Musumeci, S. A., and Puglisi-Allegra, S. (2004). Object recognition impairment in Fmrl knockout mice is reversed by amphetamine: involvement of dopamine in the medial prefrontal cortex. Behav. Pharmacol. $15,433-442$.

Vnek, N., and Rothblat, L. A. (1996). The hippocampus and long-term object memory in the rat. J. Neurosci. 16, 2780-2787.

Walker, M. P., Brakefield, T., Hobson, J.A. and Stickgold, R. (2003). Dissociable stages of human memory consolidation and reconsolidation. Nature 425 616-620.

Waltereit, R., Dammermann, B., Wulff, P., Scafidi, J., Staubli, U., Kauselmann, G.,
Bundman, M., and Kuhl, D. (2001). Arg3.1/Arc mRNA induction by $\mathrm{Ca} 2+$ and CAMP requires protein kinase $\mathrm{A}$ and mitogen-activated protein kinase/ extracellular regulated kinase activation. J. Neurosci. 21, 5484-5493.

Wang, H., Ferguson, G. D., Pineda, V. V., Cundiff, P.E., and Storm, D. R. (2004). Overexpression of type-1 adenylyl cyclase in mouse forebrain enhances recognition memory and LTP. Nat. Neurosci. 7, 635-642.

Wang, S. H., de Oliveira Alvares, L., and Nader, K. (2009). Cellular and systems mechanisms of memory strength as a constraint on auditory fear reconsolidation. Nat. Neurosci. 12, 905-912.

Winocur, G., Frankland, P.W., Sekeres, M., Fogel, S., and Moscovitch, M. (2009). Changes in context-specificity during memory reconsolidation: selective effects of hippocampal lesions. Learn. Mem. 16, 722-729.

Winters, B. D., Saksida, L. M., and Bussey, T. J. (2008). Object recognition memory: neurobiological mechanisms of encoding, consolidation and retrieval. Neurosci. Biobehav. Rev. 32 1055-1070

Winters, B. D., Tucci, M. C., and DaCostaFurtado,M. (2009). Older and stronger object memories are selectively destabilized by reactivation in the presence of new information. Learn. Mem. 16, 545-553.

Woodruff, C.C., Hayama, H. R., and Rugg, M.D. (2006). Electrophysiological dissociation of the neural correlates of recollection and familiarity. Brain Res. 1100, 125-135.

Yonelinas, A. P., Otten, L. J., Shaw, K. N., and Rugg, M.D. (2005). Separating the brain regions involved in recollection and familiarity in recognition memory. J. Neurosci. 25, 3002-3008.

Conflict of Interest Statement: The authors declare that the research was conducted in the absence of any commercial or financial relationships that could be construed as a potential conflict of interest.

Received: 03 August 2010; accepted: 29 October 2010; published online: 19 November 2010.

Citation: Davis S, Renaudineau S, Poirier $R$, Poucet B, Save E and Laroche S (2010) The formation and stability of recognition memory: what happens upon recall? Front. Behav. Neurosci. 4:177. doi: 10.3389/ fnbeh.2010.00177

Copyright (๑) 2010 Davis, Renaudineau, Poirier, Poucet, Save and Laroche. This is an open-access article subject to an exclusive license agreement between the authors and the Frontiers Research Foundation, which permits unrestricted use, distribution, and reproduction in any medium, provided the original authors and source are credited. 